\title{
Enfermedad arterial periférica y estrés oxidativo en pacientes del programa para la prevención de complicaciones de diabetes mellitus y dislipidemias
}

\section{Peripheral arterial disease and oxidative stress in patients of the program for the prevention of complications of diabetes mellitus and dyslipidemias}

\author{
Islendy Noreña-Acevedo, Cristian Camilo Peña-Quimbayo, Angélica María Ballén-Torres, \\ maría Fernanda Pineda-Corral, luz Helena Aranzález-Ramirez, \\ ISMena Vilte Ona Mockus-Sivickas • Bogotá, D.C. (Colombia)
}

DOI: https://doi.org/10.36104/amc.2019.1257

\section{Resumen}

Introducción: la enfermedad arterial periférica (EAP) es causada principalmente por aterosclerosis e intervienen la hiperglucemia y dislipidemia que desencadenan estrés oxidativo y daño vascular.

Objetivo: determinar la prevalencia de EAP en pacientes con diabetes mellitus tipo 2 (DM2) y/o prediabetes y/o dislipidemias, así como algunos factores de riesgo; también, establecer si los niveles urinarios de 8-isoprostano-f2 $\alpha$ (marcador de estrés oxidativo) están elevados en pacientes con EAP.

Diseño: estudio de tipo transversal, no probabilístico, de conveniencia.

Material y métodos: la muestra comprendió 146 pacientes con DM2 y/o prediabetes y/o dislipidemias de la Universidad Nacional de Colombia.

Se registraron factores de riesgo, síntomas relacionados con EAP, medida índice tobillo-brazo y variables bioquímicas (HbA1c\%, glucemia basal, perfil lipídico, creatinina y albuminuria). Se determinaron niveles en orina de 8-isoprostano-f2 $\alpha$ por ELISA. Los resultados de la concentración de 8-iso-PGF2 $\alpha$ /creatinuria se analizaron mediante el paquete estadístico R. La comparación de factores de riesgo se analizó mediante ANOVA/Kruskal-Wallis. Se generaron curvas ROC para analizar el poder discriminante del biomarcador. El análisis conjunto de resultados de laboratorios y de factores de riesgo se realizó mediante regresiones logísticas multivariantes.

Resultados: se evidenció prevalencia de EAP en 10 pacientes diabéticos. Como factores de riesgo se encontraron: fumar, dislipidemia, mal control metabólico, sobrepeso u obesidad. No se evidenció aumento del 8-isoprostano-f2 $\alpha$ urinario en estos sujetos.

Conclusiones: se encontró baja prevalencia de EAP en los sujetos con DM2. No se evidenció aumento del 8 -isoprostano-f $2 \alpha$ medido por ELISA en pacientes con EAP. Se recomienda ampliar el estudio con diferentes marcadores de estrés oxidativo y uso de otras técnicas. (Acta Med Colomb 2019; 44. DOI: https://doi.org/10.36104/amc.2019.1257).

Palabras clave: diabetes, dislipidemia, enfermedad arterial periférica, estrés oxidativo, 8 -isoprostano- $f 2 \alpha$.

\footnotetext{
Abstract

Introduction: peripheral arterial disease $(\mathrm{PAD})$ is mainly caused by atherosclerosis; hyperglycemia and dyslipidemia are involved and trigger oxidative stress and vascular damage.

Objective: to determine the prevalence of PAD in patients with type 2 diabetes mellitus (DM2) and / or prediabetes and / or dyslipidemias, as well as some risk factors; also, establish whether urinary levels of 8 -isoprostane-f $2 \alpha$ (oxidative stress marker) are elevated in patients with PAD.

Design: cross-sectional, non-probabilistic, convenience study.

Material and Methods: the sample included 146 patients with DM2 and / or prediabetes and / or dyslipidemias of the National University of Colombia.
}

Dra. Islendy Noreña-Acevedo: Enfermera, Magister en Fisiología. Docente Ocasional Facultad de Enfermería; Cristian Camilo Peña-Quimbayo: Estudiante de Medicina; Dra. Angélica María Ballén-Torres: Médica; María Fernanda Pineda Corral: Nutricionista; Luz Helena Aranzález-Ramírez: Bacterióloga, Magister en Bioquímica, Profesora Facultad de Medicina; Ismena Vilte Ona Mockus-Sivickas: Especialista en Endocrinología, Profesora Titular Facultad de Medicina. Universidad Nacional de Colombia, Bogotá, D.C. (Colombia). Correspondencia: Dra. Islendy Noreña Acevedo, Bogotá, D.C. (Colombia)

E-mail: inorenaa@unal.edu.co

Recibido: 31/VIII/2018 Aceptado: 22/VII/2019 
Risk factors, symptoms related to PAD, ankle-arm index measurement and biochemical variables (HbA1c\%, baseline blood glucose, lipid profile, creatinine and albuminuria) were recorded. Urine levels of 8 -isoprostane-f $2 \alpha$ were determined by ELISA. The results of the concentration of 8 -iso-PGF $2 \alpha$ / creatinuria were analyzed using the statistical package R. The comparison of risk factors was analyzed using ANOVA / Kruskal-Wallis. ROC curves were generated to analyze the discriminant power of the biomarker. The joint analysis of laboratory results and risk factors was performed using multivariate logistic regressions.

Results: prevalence of PAD was evidenced in 10 diabetic patients. Smoking, dyslipidemia, poor metabolic control, overweight or obesity were found as risk factors.There was no evidence of an increase in urinary 8 -isoprostane-f $2 \alpha$ in these subjects.

Conclusions: low prevalence of PAD was found in subjects with DM2. There was no evidence of an increase in 8 -isoprostane-f2 $\alpha$ measured by ELISA in patients with PAD. It is recommended to expand the study with different markers of oxidative stress and the use of other techniques. (Acta Med Colomb 2019; 44. DOI: https://doi.org/10.36104/amc.2019.1257).

Keywords: diabetes, dyslipidemia, peripheral arterial disease, oxidative stress, 8 -isoprostane- $f 2 \alpha$.

\section{Antecedentes}

La enfermedad arterial periférica (EAP) afecta cerca de 12 millones de personas en Estados Unidos (1). Los factores de riesgo más importantes para desarrollar la enfermedad son la diabetes mellitus tipo 2 (DM2) y el tabaquismo (2-4). La clínica de la EAP varía desde la ausencia de síntomas hasta la claudicación clásica (5-7). El diagnóstico se puede realizar mediante múltiples métodos; el más costo-efectivo es el índice tobillo-brazo (ITB) $(8,9)$. La EAP es causada principalmente por aterosclerosis, cuya patogénesis es mediada por múltiples moléculas y vías de señalización (10-12). La hiperglucemia provoca una sobreproducción de radicales libres, especies reactivas de oxígeno (ROS) y formación de productos finales de glicación avanzada que causan daño del endotelio (13-15). Además las ROS interactúan con lípidos de membranas celulares llevando a la peroxidación lipídica e incrementando la formación del 8-isoprostano-f2 $\alpha$ (8-iso-PGF2 $\alpha$ ). Las concentraciones elevadas de colesterol LDL (c-LDL) favorecen el desarrollo de la placa aterosclerótica mediante la oxidación de las LDL (LDLox) que participan en procesos que involucran la producción de ROS y la expresión de genes proinflamatorios (16). Se ha buscado medir el estrés oxidativo mediante biomarcadores; el 8-isoprostano-f2 $\alpha$ presenta la ventaja de ser cuantificable en orina casual (17).

\section{Justificación}

Los pacientes con DM2 tienen dos a tres veces más riesgo de padecer EAP (2) y ésta puede llevar a complicaciones severas como úlceras por presión y amputación (18), por esto es importante su diagnóstico temprano. No existe en Colombia, en nuestro conocimiento, un estudio sobre la prevalencia de EAP en población diabética y/o dislipidémica. Múltiples estudios han asociado la DM2 y la dislipidemia con el incremento del estrés oxidativo (19) y la $\operatorname{EAP}(20,21)$. Para medir el estrés oxidativo se han utilizado varios biomarcadores, uno de ellos, el 8-iso-PGF2 $\alpha$ (17) cuantificable en orina. Resulta de gran interés establecer en los pacientes de un programa para la prevención de las complicaciones de la diabetes mellitus y de la dislipidemia que ofrece la Facultad de Medicina de la Universidad Nacional de Colombia (equipo interdisciplinario conformado por médico, nutricionista y fisioterapeuta), la prevalencia de EAP y su relación con el control metabólico, índice de masa corporal (IMC) y estrés oxidativo determinado mediante la medición urinaria del 8 -iso-PGF2 $\alpha$. Se busca, además, identificar posibles factores de riesgo y antecedentes asociados con la presencia de EAP. La comprensión de estas relaciones podría permitir avanzar en la prevención y tratamiento de las complicaciones de los pacientes diabéticos y/o con dislipidemia.

\section{Material y Métodos}

Previa firma de consentimiento informado, se determinó: índice de masa corporal (IMC $\mathrm{Kg} / \mathrm{m}^{2}$ ), circunferencia de cintura $(\mathrm{cm})$ y presión arterial $(\mathrm{mmHg})$. Se empleó balanza OMRON®, tallímetro Health o Meter®, fonendoscopio 3M Littman $\AA$, tensiómetro Welchallyn $\AA$ y doppler Huntleigh $₫$. Se procedió a aplicar el instrumento diseñado para detectar factores de riesgo de EAP que incluía: antecedentes patológicos y familiares, hábitos (consumo de alcohol, cigarrillo y actividad física), síntomas relacionados con EAP (dolor en miembros inferiores al caminar, estar de pie o sentado, dolor o ausencia de éste al detener la caminata); síntomas atípicos (dolor en muslo o glúteo, disfunción eréctil, parestesias en extremidades), examen físico (identificación de úlceras, valoración neurológica mediante diapasón, monofilamento y martillo de reflejos) y registro de la medida del ITB, para la cual se tomó presión arterial sistólica con doppler y tensiómetro en arteria braquial y arterias pedia y tibial posterior (22). Se analizaron hemoglobina glicada (HbA1c), glucemia basal, triglicéridos, colesterol total, colesterol de alta densidad (c-HDL), colesterol de baja densidad (calculado según fórmula de Friedewald), creatinina y albuminuria, parámetros que son evaluados en cada consulta de segui- 
miento y son realizados por el laboratorio de la Unidad de Servicios de Salud de la Universidad Nacional de Colombia (Unisalud). Antes de la determinación del ITB, se recolectó la orina que se separó en 2 alícuotas que fueron almacenadas a $-80^{\circ} \mathrm{C}$ hasta su análisis para la posterior determinación de creatinuria por método colorimétrico y niveles de 8-isoPGF $2 \alpha$ por ELISA según las indicaciones de los fabricantes (Cell Biolabs).

Tipo de estudio. El diseño del estudio es de tipo transversal, no probabilístico, de conveniencia; hace uso de herramientas descriptivas para explorar el comportamiento del fenómeno, como la correlación de variables, a diversos dominios demográficos y temáticos.

Tamaño de la muestra. La muestra estudiada comprendió 146 pacientes con DM2 y/o prediabetes y/o dislipidemias pertenecientes a la Unidad de Servicios de Salud de la Universidad Nacional de Colombia (Unisalud), que participan en un programa para la prevención de las complicaciones de la Diabetes Mellitus y de las Dislipidemias que ofrece la Facultad de Medicina de la Universidad Nacional de Colombia. Los pacientes asisten periódicamente (cada tres meses) a control médico en la Facultad de Medicina.

Criterios de inclusión. Ser usuario de Unisalud, mayor de 18 años, hombres y mujeres con DM2 y/o prediabetes diagnosticadas según los criterios de la American Diabetes Association (ADA), y/o dislipidemia según los criterios de la American Association of Clinical Endocrinologists (AACE).

Criterios de exclusión. Enfermedad autoinmune activa, neoplasia activa, desórdenes psiquiátricos en tratamiento con medicación, embarazo.

Análisis estadístico. Los datos y los resultados de concentración del biomarcador (8-iso-PGF2 $\alpha /$ creatinuria) se analizaron mediante el paquete estadístico R. La comparación y el estudio de los diferentes factores de riesgo se analizó mediante ANOVA/Kruskal-Wallis. Se generaron las Curvas ROC para analizar el poder discriminante del biomarcador. Para el análisis conjunto de los diferentes resultados de los laboratorios y los factores de riesgo se llevaron a cabo regresiones logísticas multivariantes. Cuando las diferencias entre variables cumplieron el criterio de confianza del 95\% ( $\mathrm{p}<0,05)$, fueron consideradas estadísticamente significativas.

Consideraciones éticas. El presente proyecto cumplió los principios éticos para las investigaciones médicas en seres humanos establecidos por la Asociación Médica Mundial en su declaración de Helsinki. Así mismo, fue evaluado y aprobado por el Comité de Ética de la Facultad de Medicina de la Universidad Nacional de Colombia.

Resultados. Las características generales de los 146 sujetos se observan en la Tabla 1.

Tras el análisis de ITB, se encontraron 10 sujetos con ITB menor a 0.9. Estos pacientes presentaban DM2; por lo tanto, la prevalencia de EAP en los sujetos con DM2 era de 8.7\%. El 33\% de los 115 sujetos con DM2 presentaba mal control metabólico con una $\mathrm{HbA1c} \geq 7 \%$; respecto al c-LDL, $46 \%$
Tabla 1. Características generales de la población estudiada.

\begin{tabular}{|c|c|}
\hline Características generales & Número de pacientes (\%) \\
\hline Total & $146(100)$ \\
\hline Mujeres & $72(49)$ \\
\hline Hombres & $74(50)$ \\
\hline Edad 40-60 años & $15(10)$ \\
\hline Edad 60-70 años & $51(35)$ \\
\hline Edad 70-90 años & $79(54)$ \\
\hline Edad 90-100 años & $1(0.6)$ \\
\hline Diabetes mellitus 2 & $115(78)$ \\
\hline Prediabetes & $29(19)$ \\
\hline Dislipidemia & $127(86)$ \\
\hline Hipertensión arterial sistémica & $85(60)$ \\
\hline Enfermedad coronaria & $23(15)$ \\
\hline IMC $20-24.9 \mathrm{Kg} / \mathrm{m}^{2}$ & $38(26)$ \\
\hline IMC $25-29.9 \mathrm{Kg} / \mathrm{m}^{2}$ & $69(47)$ \\
\hline IMC $30-34.9 \mathrm{Kg} / \mathrm{m}^{2}$ & $31(21)$ \\
\hline IMC $35-39.9 \mathrm{Kg} / \mathrm{m}^{2}$ & $7(4)$ \\
\hline C - LDL menor $100 \mathrm{mg} / \mathrm{dL}$ & $81(55)$ \\
\hline C - LDL $100-159 \mathrm{mg} / \mathrm{dL}$ & $53(36)$ \\
\hline C - LDL $160-189 \mathrm{mg} / \mathrm{dL}$ & $10(6)$ \\
\hline
\end{tabular}

de los sujetos diabéticos se encontraba en buen control de la dislipidemia. De los sujetos con enfermedad coronaria, $21 \%$ tenía mal control metabólico $(\mathrm{HbA} 1 \mathrm{c} \geq 7 \%)$ y $17 \%$ presentaba c-LDL en metas ( $<70 \mathrm{mg} / \mathrm{dL})$. De los 146 sujetos, $47 \%$ presentó sobrepeso y $21 \%$ obesidad. Los 10 pacientes con EAP, tres mujeres y siete hombres, con edades entre 69 y 85 años, presentaron DM2 y dislipidemia. Uno de los 10 sujetos fumaba, tres presentaban claudicación intermitente, y en tres de ellos se evidenció mal control metabólico. Nueve de los sujetos con EAP tuvieron un IMC $\geq 25 \mathrm{~kg} / \mathrm{m}^{2}$. Se encontraron como factores de riesgo para EAP ser fumador, relación OR 1.51 (IC 0.18, 13); dislipidemia, OR 1.62 (IC 0.2, 13.42), mal control metabólico, OR 1.04 (IC 0.26, 4.22); se halló que tener un IMC $\geq 25 \mathrm{~kg} / \mathrm{m}^{2}$ aumenta en cuatro veces el riesgo de EAP. Por último, se encontró como factor protector para EAP, el realizar ejercicio físico con una relación OR 0.41 (IC 0.1, 1.73).

Se realizó la medición del 8-iso-PGF $2 \alpha$ en los 10 pacientes con EAP según ITB<0.9 y en 30 pacientes seleccionados de la muestra inicial quienes se clasificaron en cuatro grupos de acuerdo con control metabólico, edad y antecedente de enfermedad coronaria $(23,29)$. Los resultados se muestran en la Tabla 2.

En ninguno de los sujetos se evidenció una relación 8-isoPGF $2 \alpha$ /creatinina anormal, es decir, mayor a $0.86 \mathrm{pg} / \mathrm{mg}$. Al comparar los diferentes grupos no se encontró una diferencia estadísticamente significativa $(\mathrm{P}=0.759)$. 
Tabla 2. Resultados relación F2 -ISoPs/Creatinina ( $\mathrm{pg} / \mathrm{mg}$ ) por grupos.

\begin{tabular}{|c|c|c|c|c|c|c|c|}
\hline & & \multicolumn{6}{|c|}{ Grupos } \\
\hline & & $\begin{array}{l}\text { Grupo } 1 \\
\text { con EAP }\end{array}$ & $\begin{array}{c}\text { Grupo 2 } \\
\text { Mal control } \\
\text { metabólico, } \\
\text { no enfermedad } \\
\text { coronaria }>65 \\
\text { años }\end{array}$ & $\begin{array}{c}\text { Grupo } 3 \\
\text { Mal control } \\
\text { metabólico, } \\
\text { no enfermedad } \\
\text { coronaria }<65 \\
\text { años }\end{array}$ & $\begin{array}{c}\text { Grupo } 4 \\
\text { Buen control } \\
\text { metabólico, } \\
\text { no enfermedad } \\
\text { coronaria }>65 \\
\text { años }\end{array}$ & $\begin{array}{c}\text { Grupo 5 } \\
\text { Buen control } \\
\text { metabólico, } \\
\text { enfermedad } \\
\text { coronaria }>65 \\
\text { años }\end{array}$ & Subtotal \\
\hline \multirow{7}{*}{$\begin{array}{l}\text { Relación } \\
\text { F2 -ISoPs/ } \\
\text { creatinina } \\
\text { (pg/mg) }\end{array}$} & Media & 0.0102 & 0.0241 & 0.0159 & 0.0209 & 0.0029 & 0.0148 \\
\hline & Desviación estándar & 0.0273 & 0.0579 & 0.0351 & 0.0497 & 0.0033 & 0.0383 \\
\hline & Máximo & 0.088 & 0.1671 & 0.0953 & 0.1434 & 0.0098 & 0.1671 \\
\hline & Percentil 95 & 0.088 & 0.1671 & 0.0953 & 0.1434 & 0.0098 & 0.1193 \\
\hline & Mediana & 0.0013 & 0.0023 & 0.0022 & 0.0017 & 0.0013 & 0.0018 \\
\hline & Percentil 05 & 0.0005 & 0.0009 & 0.001 & 0.0009 & 0.0007 & 0.0007 \\
\hline & Mínimo & 0.0005 & 0.0009 & 0.001 & 0.0009 & 0.0007 & 0.0005 \\
\hline
\end{tabular}

Se realizó la comparación del grupo 4 (sujetos con buen control metabólico, sin enfermedad coronaria y sin EAP) contra los otros grupos sin encontrar diferencias significativas (Tabla 3 ).

\section{Discusión}

La prevalencia de enfermedad arterial periférica hallada es menor a la descrita en la población norteamericana, australiana, belga, costarricense y mexicana $(1,25-28)$ no hay datos de prevalencia en Colombia, sin embargo el presente puede considerarse un estudio piloto (29). Los pacientes del estudio asisten a control médico periódico, lo que facilita la identificación de limitantes que dificultan la adherencia al manejo y el control metabólico; por tanto, la prevalencia de EAP podría ser menor a la de otros grupos sin seguimiento regular. El 67\% de los pacientes diabéticos del estudio se encontraba en metas de $\mathrm{HbA} 1 \mathrm{c}$. Lo anterior está de acuerdo con el estudio UKPDS (30) en el que un buen control glucémico está asociado con disminución del riesgo de complicaciones macrovasculares; de igual manera una revisión sistemática y metaanálisis de la Universidad de Berna encontró que un mejor control glucémico en pacientes con DM1 y DM2 se asocia con una disminución de la incidencia de eventos macrovasculares especialmente EAP y accidentes cerebrovasculares (31). El consumo de cigarrillo estuvo presente en los pacientes con EAP, no se indagó por la intensidad ni duración del consumo pero se ha encontrado que la prevalencia e incidencia de EAP es mayor en pacientes con alto consumo de tabaco y tiempo prolongado de exposición (32-35). La mayoría de los pacientes con EAP tenían un IMC $\geq 25$. En un estudio con población china, se observó que el incremento del IMC se relacionó en forma directa con la presencia de EAP (35). Los pacientes con obesidad evidencian niveles elevados de triglicéridos y de ácidos grasos libres circulantes, lo que se ha asociado con aumento de la producción de radicales libres (37). En un grupo de pacientes diabéticos obesos de Irán, se evidenció aumento de la concentración plasmática del 8-isoPGF $2 \alpha$ medido por ELISA (38). Todos los pacientes con EAP
Tabla 3. Significancia estadística de la comparación entre grupos de la relación F2 -ISoPs/ creatinina ( $\mathrm{pg} / \mathrm{mg})$

\begin{tabular}{|c|c|c|}
\hline \multicolumn{2}{|c|}{ Grupos comparados } & P. Valor \\
\hline Grupo 4 & Grupo 1 & 0.3 \\
\hline Grupo 4 & Grupo 2 & 0.453 \\
\hline Grupo 4 & Grupo 3 & 0.412 \\
\hline Grupo 4 & Grupo 5 & 0.171 \\
\hline
\end{tabular}

de este estudio presentaron DM2 y dislipidemia.La DM2 y las dislipidemias incrementan el estrés oxidativo y aumentan el riesgo de EAP $(11,12,39)$. Se midió el 8-iso-PGF2 $\alpha$, el más abundante de su clase y considerado buen marcador biológico del estrés oxidativo, además con la ventaja de ser cuantificable en una muestra de orina. No se encontró aumento del 8 -iso-PGF $2 \alpha$ en los pacientes con EAP y no se evidenciaron diferencias estadísticamente significativas con los otros grupos. Varios factores podrían explicar este resultado: se empleó ELISA para su cuantificación mientras que otros estudios han utilizado la cromatografía líquida o de gases acoplada a espectrometría de masas $(40,41)$, además las concentraciones urinarias pueden ser diferentes a las plasmáticas, ya que en orina sólo se miden las formas libres (42).

\section{Conclusiones}

Se encontró una baja prevalencia de EAPen los pacientes con DM2 del programa para la prevención de las complicaciones de la diabetes mellitus tipo 2 y de las dislipidemias que ofrece la Facultad de Medicina de una universidad colombiana. Esta baja prevalencia podría deberse al control periódico de los pacientes. El seguimiento regular por un grupo interdisciplinario conformado por médico, nutricionista y fisioterapeuta parece tener un efecto favorable sobre los factores de riesgo modificables. No se evidenció aumento de los niveles urinarios de un marcador de estrés oxidativo, el 8-isoprostano-f $2 \alpha$ medido por ELISA en los pacientes con EAP. Se recomienda ampliar el estudio con otros marcadores 
de estrés oxidativo y el uso de técnicas como la cromatografía líquida o de gases acoplada a espectrometría de masas.

\section{Referencias}

1. Selvin E, Erlinger TP. Prevalence of and risk factors for peripheral arterial disease in the United States: Results from the National Health and Nutrition Examination Survey, 1999-2000. Circulation [Internet]. 2004 [citado el 15 de mayo de 2018] ;110(6):738-743. Disponible en: https://www.ahajournals. org/doi/10.1161/01.CIR.0000137913.26087.F0?url_ver=Z39.88-2003\&rfr_ id=ori\%3Arid\%3Acrossref.org\&rfr_dat=cr_pub\%3Dpubmed\&

2. Thiruvoipati T, Kielhorn CE, Armstrong EJ. Peripheral artery disease in patients with diabetes: Epidemiology, mechanisms, and outcomes. World J Diabetes. 2015;6(7):961-969

3. Criqui MH, Aboyans V. Epidemiology of Peripheral Artery Disease. Circ Res [Internet]. 2015 [citado el 17 de mayo de 2018];116(9):1509-1526. Disponible en: https://www.ahajournals.org/doi/abs/10.1161/circresaha.116.303849

4. Malone M, Lau NS, White J, et al. The effect of diabetes mellitus on costs and length of stay in patients with peripheral arterial disease undergoing vascular surgery. Eur J Vasc Endovasc Surg. 2014;48(4):447-451

5. Bailey MA, Griffin KJ,Scott DJA. Clinical Assessment of Patients with Peripheral Arterial Disease. Seminars in Interventional Radiology [Internet]. 2014 [citado el 2 de junio de 2018]; 31(4):292-299. Disponible en: https://www.ncbi.nlm.nih.gov/ pmc/articles/PMC4232424/

6. Khawaja FJ, Kullo IJ. Novel markers of peripheral arterial disease. Vasc Med. 2009;14(4):381-392

7. Mohler ER. Therapy insight: peripheral arterial disease and diabetes - frompathogenesis to treatment guidelines. Nat Clin Pract Cardiovasc Med. 2007;4(3):151-162.

8. Solomon CG, Kullo IJ, Rooke TW. Peripheral Artery Disease. $N$ Engl J Med. 2016;374(9):861-87.

9. Bluro, Ignacio M.Consenso de enfermedad vascular periférica: Versión resumida. Rev. argent. Cardiol [Internet].2015 [citado el 14 de julio de 2018]; 83(5), 461-486. Disponible en: http://www.scielo.org.ar/scielo.php?script=sci_arttext\&pid=S1850$37482015000500021 \& \operatorname{lng}=$ es\&tlng=es.

10. Krishna S, Moxon J, Golledge J. A review of the pathophysiology and potential biomarkers for peripheral artery disease. Int J Mol Sci [Internet]. 2015 [citado el 10 de junio de 2018];16(5):11294-11322. Disponible en: https://www.ncbi.nlm. nih.gov/pmc/articles/PMC4463701/

11. Sena CM, Pereira AM, Seia R. Endothelial dysfunction - A major mediator of Diabetic vascular disease. Biochim Biophys Acta - Mol Basis Dis [Internet]. 2013 [citado el 10 de junio de 2018];1832(12):2216-2231. Disponible en: https://www. sciencedirect.com/science/article/pii/S0925443913002718?via\%3Dihub

12. Brownlee M. The pathobiology of diabetic complications: A unifying mechanism. Diabetes [Internet]. 2005 [citado el 10 de junio de 2018];54(6):1615-1625. Disponible en: http://diabetes.diabetesjournals.org/content/54/6/1615.long

13. Chilelli NC, Burlina S, Lapolla A. AGEs, rather than hyperglycemia, are responsible for microvascular complications in diabetes: A "glycoxidation-centric" point of view. Nutr. Metab. Cardiovasc. Dis. 2013;23(10):913-919.

14. Lubrano V, Balzan S. LOX-1 and ROS, inseparable factors in the process of endothelial damage. Free Radic Res. 2014;48(8):841-848

15. Nowotny K, Jung T, Höhn A, Weber D, Grune T. Advanced glycation end products and oxidative stress in type 2 diabetes mellitus. Biomolecules [Internet]. 2015 [citado el 11 de junio de 2018];5(1):194-222. Disponible en: https://www. ncbi.nlm.nih.gov/pmc/articles/PMC4384119/

16. Perrotta I, Aquila S. The role of oxidative stress and autophagy in atherosclerosis. Oxid Med Cell Longev [Internet]. 2015 [citado el 12 de junio de 2018]; 1-10. Disponible en: https://www.hindawi.com/journals/omcl/2015/130315/

17. Basu, S. F2-Isoprostanes in Human Health and Diseases: From Molecular Mechanisms to Clinical Implications. Antioxidants \& Redox Signaling.2008; 10(8), 1405-1434.

18. Lau JF, Weinberg MD, Olin JW. Peripheral artery disease. Part 1: clinical evaluation and noninvasive diagnosis. Nat Rev Cardiol. 2011;8(7):405-418.

19. Roberts CK, Won D, Pruthi S, Lin SS, Barnard RJ. Effect of a diet and exercise intervention on oxidative stress, inflammation and monocyte adhesion in diabetic men. Diabetes Res Clin Pract. 2006;73(3):249-259

20. Dichi I, Wadner J, Colado Simao AN. Dyslipidemia and atherosclerosis. In: Role of Oxidative Stress and Chronic Diseases. Primera edición. CRC Press; 2014. p. 139-141

21. Dichi I, Wadner J, Colado Simao AN. Dyslipidemia and atherosclerosis. In: Role of Oxidative Stress and Chronic Diseases. First. CRC Press; 2014. p. 137-139

22. Gerhard-Herman MD, Gornik HL, Barrett C, Barshes NR, Corriere MA, Drachman DE, et al. AHA/ACC guideline on the management of patients with lower extremity peripheral artery disease: executive summary: a report of the american college of cardiology/american heart association task force on clinical practice guidelines. In: Circulation. 2016; 135:e686-e725.
23. Perrone $\mathbf{P}$, Chiariello $\mathbf{M}$. Coronary artery disease and intermittent claudication: how to manage the patient. European Heart Journal Supplements [Internet]. 2002 [citado el 20 de junio de 2018]; 4, 58-62. Disponible en: https://academic.oup.com/ eurheartjsupp/article/4/suppl_B/B58/470623

24. Munzel T,Gori T, Bruno R, Taddei S. Is oxidative stress a therapeutic target in cardiovascular disease? European Heart Journal. 2010; 31, 2741-2749.

25. Norman PE, Eikelboom JW, Hankey GJ. Peripheral arterial disease: Prognostic significance and prevention of atherothrombotic complications. Med J Aust. 2004;181(3):150-154.

26. Bergiers S, Vaes B, Degryse J. To screen or not to screen for peripheral arterial disease in subjects aged 80 and over in primary health care: a cross-sectional analysis from the BELFRAIL study. BMC Fam Pract. 2011;12(39):39.

27. Quiróz Meza G, Salazar Nassar J, Castillo Rivas J. Atención de los pacientes con enfermedad arterial periférica en los hospitales de la Caja Costarricense de Seguro Social. Acta Médica Costarricense [Internet]. 2011[citado el 10 de julio de 2018]; 53 (4): 182-187. Disponible en:<http://www.redalyc.org/articulo. oa?id=43421508005 $>$ ISSN 0001-6002

28. Diaz Cruz G, Velasquez Tlapanco J, Islas Cortés B, Martinez Martinez M, Camacho Calderón N, Gallardo Vidal L. Prevalencia de enfermedad arterial periférica con base al índice tobillo / brazo en pacientes con diabetes mellitus tipo 2 . European Scientific Journal [Internet]. 2014 [citado el 6 de julio de 2018];10(30):922. Disponible en: http://eujournal.org/index.php/esj/article/viewFile/4438/4247.

29. Restrepo L, Hernández N, Henao J, Cadavid L, Jaramillo S, Aguirre D. Tratamiento de la enfermedad arterial periférica de las extremidades inferiores con células mononucleares de médula ósea autólogas reporte de seguimiento a un año.Medellín. Iatreia 2012. Vol. 25.p. 323-333.

30. UK Prospective Diabetes Study Group. Tight blood pressure control and risk of macrovascular and microvascular complications in type 2 diabetes: UKPDS 38. $B M J$ [Internet]. 1998 [citado el 21 de junio de 2018];317(7160):703-713. Disponible en: https://www.ncbi.nlm.nih.gov/pmc/articles/PMC28659/

31. Stletter C, Allemann S, Jüni P, Cull C, Holman R, Egger M, Krähenbühl, Diem P. Glycemic control and macrovascular disease in types 1 and 2 diabetes mellitus: Meta-analysis of randomized trials. American Heart Journal. 2006; 152 (1) 27-38.

32. Willigendael E, Joep A, Bartelink M, Barthold K,Boiten J, Moll F, Büller H, Prins M. Influence of smoking on incidence and prevalence of peripheral arterial disease. Journal of vascular surgery [Internet]. 2004 [citado el 30 de junio de 2018]; 40 (6): 1158-1165. Disponible en: https://www.sciencedirect.com/science/article/ pii/S0741521404011413

33. Ngu N, McEvoy M. Environmental tobacco smoke and peripheral arterial disease: A review. Atherosclerosis. 2017; 266: 113-120.

34. Pasupathi1 P, Saravanan G,Farook J,Oxidative Stress Bio Markers and Antioxidant Status in Cigarette Smoker Compared to Nonsmokers. J. Pharm. Sci. \& Res [Internet].2009 [citado el 1 de julio de 2018]; 1(2): 55-62.Disponible en: http:// citeseerx.ist.psu.edu/viewdoc/download?doi=10.1.1.180.3933\&rep=rep1\&type= pdf

35. Lu J, Creager M. The Relationship of Cigarette Smoking to Peripheral Arterial Disease. Rev Cardiovasc Med. 2004; 5 (4): 189-192.

36. Huang Y, Min X, Lan X, Tiange W, Huang X, Xiaofei Lv, Chen Y, Ding L, Lin L, Wang W, Yufang B, Yimin S, Zhang Y, Guang N. Obesity and peripheral arterial disease: A Mendelian Randomization analysis. Atherosclerosis.2016; (247) 218-224.

37. Vincent H, Taylor A. Biomarkers and potential mechanisms of obesity-induced oxidant stress in humans. International Journal of Obesity [Internet]. 2006 [citado el 4 de julio de 2018]; 30: 400-418.

38. Zamania E, Djalalia M, Eshraghianb M, Sotoudehc G, Sadrzadeh-Yeganehc H, Koohdanid F. Association of body mass index with oxidative stress in patients with Type 2 diabetes: do apolipoprotein A-II $-265 \mathrm{~T}>\mathrm{C}$ polymorphism alter this association?. JNSD. 2015;1 (3): 127-133.

39. Shi Y, Vanhoutte PM. Macro-and Microvascular Endothelial Dysfunction in Diabetes. J. Diabetes. 2017;9(5):434-449.

40. Griendling K,Touyz R, Zweier J, Dikalov S, Chilian W, Chen Y, Harrison D, Bhatnagar A. Measurement of Reactive Oxygen Species, Reactive Nitrogen Species, and Redox-Dependent Signaling in the Cardiovascular System. Circ Res. 2016; 119(5):39-75.

41. Anderson C, Milne GL, Park YM, Sandler DP, Nichols HB. Dietary Glycemic Index and Glycemic Load Are Positively Associated with Oxidative Stress among Premenopausal Women. J Nutr. 2018 Jan 1;148(1):125-130. Disponible en: doi: 10.1093/jn/nxx022.

42.Zhang $\mathbf{Z}$. Systematic review on the association between F2-isoprostanes and cardiovascular disease. Ann Clin Biochem [Internet]. 2013 [citado el 4 de julio de 2018]; 50: 108-114.Disponible en: http://journals.sagepub.com/doi/pdf/10.1258/ acb.2012.011263 